\title{
Direct Online Monitoring of E-caprolactone Polymerisation via Dielectric Property Measurement
}

\author{
Alexis N. Kalamiotis ${ }^{1}$, Alexander A. Ilchev ${ }^{1}$, Derek J. Irvine ${ }^{1}$, Georgios A. Dimitrakis ${ }^{1}$ \\ ${ }^{1}$ Microwave Process Engineering Group (MPE), \\ Department of Chemical and Environmental Engineering, \\ University of Nottingham, Nottingham, NG7 2RD, UK \\ E-mail: alexis.kalamiotis@nottingham.ac.uk
}

\begin{abstract}
The use of dielectric spectroscopy to monitor the progress of the ring opening polymerisation of $\varepsilon$-caprolactone in real time is demonstrated. A coaxial probe sensor was used to monitor the dielectric properties during the polymerisation progress and samples were extracted at set times for off-line analysis with Nuclear Magnetic Resonance spectroscopy. It was observed that the dielectric properties can be linearly related with the conversion of monomer to polymer. Furthermore, the analysis of the dielectric data with frequency enabled the identification of the most suitable strategy for the online monitoring of the polymerisation. As a result, the online measurement of the dielectric properties can be used to identify in real time key reaction parameters such as monomer to polymer conversion. This could lead to improved product quality and batch to batch repeatability by identifying the reaction end-point to achieve a target conversion.
\end{abstract}

Key words: online reaction monitoring, ring opening polymerisation, controlled polymerisation, dielectric properties, dielectric spectroscopy

\section{Introduction}

In the last few decades the synthesis of polymers has been evolved and new techniques have been developed such as the Nitroxide Mediated Polymerization, [1] Atom Transfer Radical Polymerization [2] and RingOpening Polymerisation. [3] These techniques allow us to control the molecular architecture of produced polymers. However, the lack of real time feedback on reaction progress can led to batch to batch variability in larger scale products. Most of existing characterisation techniques require sample extraction and preparation. As a result, they cannot be used to monitor the reaction progress in real time. [4]

The aim of this study is to monitor the progress of the ring-opening polymerisation of $\varepsilon$-caprolactone $(\mathrm{CL})$ via the online measurement of the systems dielectric properties (DPys) using a coaxial probe sensor. The dielectric properties consist of the dielectric constant $\left(\varepsilon^{\prime}\right)$, which expresses the ability of a material to store energy through polarisation, and the dielectric loss $\left(\varepsilon^{\prime \prime}\right)$ which denotes the ability of the material to dissipate the stored energy into heat. DPys are frequency and temperature dependent. [3]

\section{Experimental}

\subsection{Materials}

E-caprolactone (99\%), tin(II) 2-ethylhexanoate $(96 \%)\left(\mathrm{Sn}(\mathrm{Oct})_{2}\right)$ and benzyl alcohol (99\%) $(\mathrm{BzOH})$ were purchased from Alfa Aesar and used as received without any purification.

\subsection{Ring Opening Polymerisation Procedure}

All experiments were conducted with a constant $[\mathrm{CL}]:\left[\mathrm{Sn}(\mathrm{Oct})_{2}\right]:[\mathrm{BzOH}]$ molar ratio of $800: 1: 10$. The reaction mixture was placed into a $50 \mathrm{~mL}$ flask, pre-purged with nitrogen, and heated to the target reaction temperature $\left(160{ }^{\circ} \mathrm{C}\right)$ with the use of a preheated oil bath. The mixture was mechanically stirred throughout the reaction progress and samples were extracted at set times for off-line analysis. Additionally, at the same time an online measurement of the DPys was performed.

\subsection{On-line Dielectric Characterization Method}

The determination of the dielectric response of the reaction mixture was conducted with the use of an Agilent 85070E open ended coaxial probe sensor and a Keysight N5232A PNA-L Vector Network Analyser (VNA). The probe was 
immersed into the reaction mixture and a swept signal $(0.5 \mathrm{GHz}-20 \mathrm{GHz})$ transmitted from the VNA, through the probe, into the reaction mixture. Depending on the DPys of the sample, a portion of that signal was reflected back to the VNA and the dielectric properties were calculated using Agilent's provided software.

\subsection{Off-line Molecular Analysis}

Samples were extracted at set times during the polymerisation for off-line analysis with Nuclear Magnetic Resonance spectroscopy (NMR) to calculate the mole ratio between monomer and polymer. ${ }^{1} \mathrm{H}$ NMR spectra were recorded in deuterated chloroform on a Bruker AVIIIHD 400 spectrometer with cryoprobe. Conversion was determined by comparing the integral of the proton resonance of the methylene moiety adjacent to oxygen of the carbonyl group for both the monomer and polymer.

\section{Results and Discussion}

It was observed that both $\varepsilon^{\prime}$ and $\varepsilon^{\prime \prime}$ decreased during the polymerisation across the frequency range investigated $(0.5 \mathrm{GHz}-20 \mathrm{GHz})$. The largest scale measurement changes for $\varepsilon^{\prime}$ and $\varepsilon^{\prime \prime}$ were found at frequencies $0.95 \mathrm{GHz}$ and 18.35 GHz respectively. Fig. 1 and Fig. 2 show the trend on the $\varepsilon^{\prime}$ and $\varepsilon^{\prime \prime}$ at frequency $0.95 \mathrm{GHz}$ and $18.35 \mathrm{GHz}$ as the conversion ranges from $31.75 \%$ to $98.69 \%$. Each data point represents the average of three measurements and the error bars show the standard deviation.

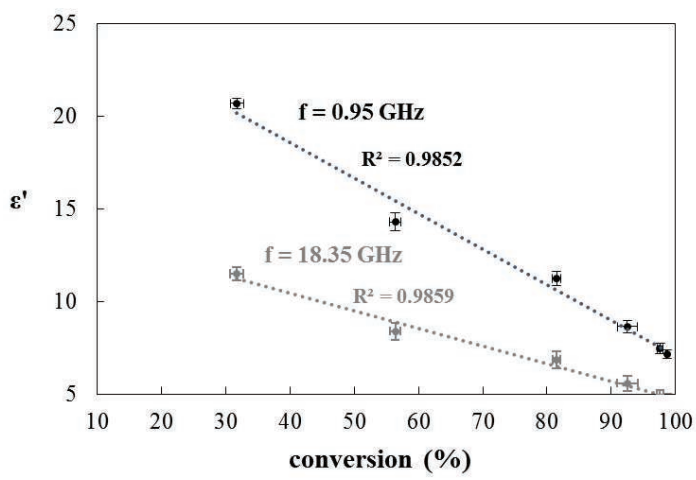

Fig. 1. Graph of dielectric constant ( $\left.\varepsilon^{\prime}\right)$ versus conversion for frequency $0.95 \mathrm{GHz}$ and $18.35 \mathrm{GHz}$.

Fig. 1 and Fig. 2 denote that both $\varepsilon^{\prime}$ and $\varepsilon^{\prime \prime}$ can be linearly related to conversion with high coefficient of determination $\left(R^{2}\right)$ values. The optimum frequency to monitor conversion depends on the type of sensor for the online measurement of the dielectric properties. Contact methods such as the coaxial probe achieve high accuracy for high loss values. The $\varepsilon^{\prime \prime}$ data at high frequencies, such as $18.35 \mathrm{GHz}$, exhibited high values (see Fig. 2). Resonator methods are accurate for measuring low loss materials and consequently low frequencies, such as $0.95 \mathrm{GHz}$, will provide low loss values.
However, the high $\varepsilon^{\prime}$ values at low frequencies (see Fig. 1) will affect the sensitivity of the monitoring system if a resonator method is the selected type of sensor.

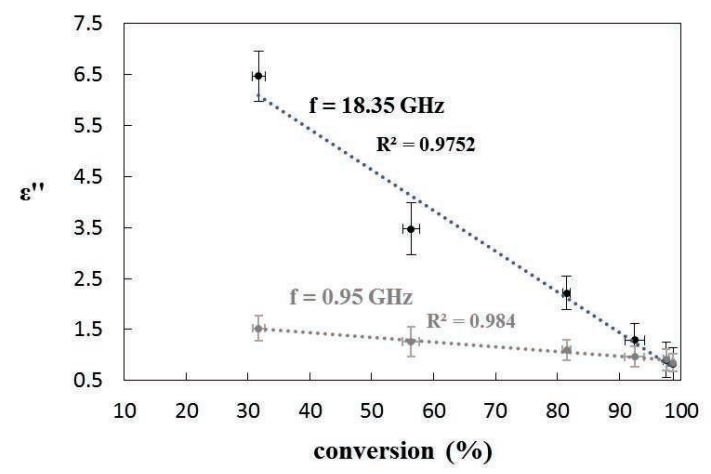

Fig. 2. Graph of dielectric loss $\left(\varepsilon^{\prime \prime}\right)$ versus conversion for frequency $0.95 \mathrm{GHz}$ and $18.35 \mathrm{GHz}$.

\section{Conclusions}

This study has shown that the online measurement of the dielectric properties during the $\varepsilon$-caprolactone polymerisation can be used to identify key reaction parameters such as monomer to polymer conversion. The analysis of the dielectric data with frequency allowed the identification of the most suitable monitoring strategy by comparing the dielectric data at low and high frequencies. It was observed that low frequencies provide low loss values and, as a result, resonant methods are more accurate. High frequencies provide high loss values and a contact method such as the coaxial probe is the most suitable type of sensor. The proposed method is non-destructive, does not require sampling and can be implemented in most polymerisation reactor geometries. The application of this method can optimise product quality and batch to batch repeatability by identifying the optimum point of termination of the reaction to achieve the target conversion.

\section{References}

[1] C. J. Hawker, A. W. Bosman, E. Harth, New Polymer Synthesis by Nitroxide Mediated Living Radical Polymerizations, Chemical Reviews 101 (12), 3661-3688 (2001); doi: 10.1021/cr990119u

[2] K. Matyjaszewski, J. Xia, Atom Transfer Radical Polymerization, Chemical Reviews 101 (9), 29212990 (2001); doi: 10.1021/cr940534g

[3] M. J. Kamaruddin, N. T.Nguyen, G. A. Dimitrakis, J. El harfi, E. R. Binner. S. W. Kingman, E. Lester, J. P. Robinson, D. J. Irvine, Continuous and direct 'in situ' reaction monitoring of chemical reactions via dielectric property measurement: controlled polymerisation, RSC Advances 4, 5709-5717 (2014); doi: 10.1039/C3RA46941G

[4] M. Destarac, Controlled Radical Polymerization: Industrial Stakes, Obstacles and Achievements, Macromolecular Reaction Engineering 4 (3-4), 165-179 (2010); doi: 10.1002/mren.200900087 